山्山FANÇAISE

$>\mathrm{DE}$

$\stackrel{1=1}{\simeq}$ PÉDAGOGIE
Revue française de pédagogie

Recherches en éducation

164 | juillet-septembre 2008

Évaluation des politiques éducatives et comparaisons internationales

\title{
PeYRONIE Henry et Vergnioux Alain (dir.). Éducation et longue durée. Actes du colloque de Cerisy-la-Salle (22-26 septembre 2005)
}

Caen : Presses universitaires de Caen, 2007. - 218 p.

Jean-Marc Lamarre

\section{(2) OpenEdition \\ Journals}

Édition électronique

URL : http://journals.openedition.org/rfp/2079

DOI : $10.4000 / \mathrm{rfp} .2079$

ISSN : $2105-2913$

Éditeur

ENS Éditions

Édition imprimée

Date de publication : 1 juillet 2008

Pagination : 174-177

ISBN : 978-2-7342-1136-5

ISSN : 0556-7807

\section{Référence électronique}

Jean-Marc Lamarre, "PeYronie Henry et Vergnioux Alain (dir.). Éducation et longue durée. Actes du colloque de Cerisy-la-Salle (22-26 septembre 2005) », Revue française de pédagogie [En ligne], 164 | juillet-septembre 2008, mis en ligne le 14 octobre 2010, consulté le 21 décembre 2020. URL : http:// journals.openedition.org/rfp/2079; DOI : https://doi.org/10.4000/rfp.2079

Ce document a été généré automatiquement le 21 décembre 2020.

(C) tous droits réservés 


\section{PEYRONIE Henry et VERGNIOUX Alain (dir.). Éducation et longue durée. Actes du colloque de Cerisy-la-Salle (22-26 septembre 2005)}

Caen : Presses universitaires de Caen, 2007. - 218 p.

Jean-Marc Lamarre

\section{RÉFÉRENCE}

Peyronie Henry et Vergnioux Alain (dir.). Éducation et longue durée. Actes du colloque de Cerisy-la-Salle (22-26 septembre 2005). Caen : Presses universitaires de Caen, 2007. $218 \mathrm{p}$.

1 Cet ouvrage rassemble des études sur l'école française dans la perspective de la longue durée. Les chercheurs réunis au Centre culturel de Cerisy-la-Salle à l'initiative du CERSE de l'Université de Caen relèvent de disciplines différentes dans le champ des sciences de l'éducation (philosophie, anthropologie, histoire, sociologie). Ce qui fait l'unité du livre, c'est la problématisation des phénomènes scolaires en termes de longue durée. Ce choix d'un "grand angle» s'avère fécond pour comprendre l'école, en tant qu' « institution centrale de la modernité classique» (Tarot, p. 24). L'ouvrage permet en effet de prendre la mesure de la complexité de cette modernité scolaire empêtrée dans des temporalités multiples, de dépasser certains faux débats et de s'interroger sur l'école d'après la modernité classique.

2 Dans un texte introductif, $C$. Tarot thématise comme tel le concept de longue durée appliqué à l'école. Si la longue durée évoque d'abord une sorte d'inertie, elle contient aussi des potentialités de créativité. Les différentes communications dans ce colloque montrent bien cet enchevêtrement compliqué d'inerties et de changements, de ruptures et de continuités. C. Tarot distingue trois modalités (courte, moyenne, longue) 
de la longue durée, l'histoire de l'école étant faite du métissage de ces durées hétérogènes. La longue durée courte est celle de l'institution républicaine depuis la Révolution française. La «longue durée moyenne, c'est le rapport de l'École à l'humanisme et peut-être sa fin.» (p. 22). La culture humaniste inscrivait l'école républicaine dans la durée moyennement longue des grands héritages classiques; or, remarque $\mathrm{C}$. Tarot, la transmission de cet héritage est devenue quasi impossible. Le troisième niveau de longue durée est celui du rapport de l'école à l'écrit. L'école, en tant qu'institution de l'écrit, est aujourd'hui confrontée au défi de la culture de masse qui repose sur l'image et l'oralité et à celui d'Internet et des techniques interactives de communication. Elle est prise dans la tension entre le temps long de la transmission et de l'apprentissage et le temps court de la société avec lequel l'impératif d'adaptation la contraint à se synchroniser.

Nous allons rendre compte de l'ouvrage en regroupant les textes selon qu'ils se situent dans la dimension longue, moyenne ou courte de la longue durée. Un premier ensemble de textes aborde l'éducation dans la dimension de la très longue durée (Bugnard, Fabre, Clanché). P.-P. Bugnard étudie, des cathédrales médiévales aux capitales européennes, un temps où, avant l'alphabétisation de masse, la transmission opérait par des « jeux d'espace » (p. 41). Il repère dans ce temps long une rupture entre le sacré et le profane. L'espace pédagogique est d'abord, avec la cathédrale, un espace sonore et iconique orienté dans une perspective eschatologique vers le levant et où retentit la parole qui transmet, hors du cadre de l'écrit, le message sacré. « Le programme grégorien prépare ainsi un socle pédagogique extrêmement résistant: le primat de la récitation, de la circulation de la connaissance, en cycles annuels, en un lieu délimité. Habitus résistant de la pédagogie frontale, la croyance en l'opérationnalisation du discours oral dérive-telle de ce vieux socle dont le principe de propagation viva voce aura survécu à son corpus?» (p. 43). Puis l'espace pédagogique devient, avec les capitales européennes à partir du $\mathrm{XVII}^{\mathrm{e}}$ siècle, un espace profane orienté (ou plutôt "occidenté») vers le couchant dans une perspective hygiéniste et ségrégative; et la "ségrégation socialepédagogique est-ouest des capitales européennes» (p.49) va s'étendre de l'espace urbain à l'espace scolaire. Cette approche par la très longue durée apporte un éclairage généalogique nouveau sur la structure ségrégative de l'école en deux ordres (que l'étude d'H. Peyronie sur la coupure primaire-secondaire complète du point de vue d'une durée beaucoup plus courte). Dans sa communication, $C$. Tarot montre que c'est en tant qu'institution de l'écrit que l'école est dans la longue durée ; P.-P. Bugnard met en évidence que le mode oral de transmission constitue aussi une tradition longue qui survit aux savoirs sacrés et résiste à l'alphabétisation.

4 M. Fabre, dans un travail de "généalogie de nos valeurs éducatives » (p. 53), qui prend appui sur L'Évolution pédagogique en France (1), remonte également au haut Moyen Âge et voit aussi dans l'histoire longue de l'école un passage du sacré au profane. Durkheim repère dans la longue durée un idéal éducatif qui perdure en profondeur malgré les changements de surface et qui a son origine ni dans la paideia antique ni dans l'humanisme de la Renaissance mais dans les écoles carolingiennes du viII e siècle. Selon lui, c'est le christianisme médiéval qui introduit dans nos institutions éducatives les valeurs d'unité de l'enseignement, d'intériorité et de conversion. L'histoire longue de l'école est un processus de sécularisation : « Notre conception du but s'est sécularisé ; par suite les moyens employés doivent changer eux-mêmes, mais le schéma abstrait du processus éducatif n'a pas varié. Il s'agit toujours de descendre dans ces profondeurs de 
l'âme dont l'Antiquité n'avait pas conscience." (cité p.60). Durkheim, comme le montre M. Fabre, repère dans l'histoire pédagogique des défaillances (le formalisme, l'esthétisme, l'utilitarisme); celles-ci ne sont pas de simples accidents mais «des tendances lourdes qui semblent toujours faire obstacle à la réalisation du projet normatif de l'école de la modernité. » (p. 61). La communication de M. Fabre montre non seulement l'intérêt de la pensée de Durkheim (en particulier l'idée de sécularisation de l'idéal éducatif) mais aussi la pertinence de la perspective de la longue durée qui, par la prise de recul, permet de nous interroger sur notre présent. M. Fabre fait ainsi l'hypothèse qu'avec le postmodernisme « un deuxième seuil de sécularisation » (p. 66) est franchi. « Il s'agit de savoir si l'école d'aujourd'hui peut et doit rester fidèle à l'idéal éducatif de la chrétienté ou si elle doit désormais en chercher un autre dans la mesure où les idées même d'unité, de visée encyclopédique, de conversion, s'avèrent aujourd'hui plus difficiles à appréhender que jamais. » (p. 66).

P. Clanché s'interroge sur les relations problématiques entre l'école et la culture vivante traditionnelle kanake. La perspective de la longue durée lui permet de résoudre le problème de la place de l'école kanake, en tant que celle-ci est à la fois « revendiquée et déjetée » (p.115). En effet, bien que ce soient les autorités coutumières qui aient demandé l'implantation d'écoles publiques dans les tribus, ces écoles sont excentrées et rejetées en périphérie, dans des zones limites (en lisière de forêt ou près de cimetières). Cet emplacement des écoles est révélateur de la place faite aux savoirs, perçus comme "savoirs européens" ou "savoirs des Blancs ». "Comme les morts qu'il convient de respecter mais dont il faut aussi se méfier, il faut respecter officiellement le savoir considéré dans sa généralité abstraite, mais aussi s'en défier. Comme les morts, les savoirs peuvent être dangereux» (p.111). Par rapport à la très longue durée de la culture kanake, « l'école ne constitue pas un lieu officiel et reconnu de socialisation et d'apprentissage culturels. » (p. 105).

6 E. Prairat fait le choix de la modalité moyenne de la longue durée. La punition, en tant que rapport de pouvoir sur le corps, est permanente dans l'éducation et relève en ce sens de la très longue durée anthropologique. Dans un premier travail d'inspiration foucaldienne, E. Prairat a commencé par mettre à jour quatre grandes formes anthropologiques de punition et d'investissement du corps (la punition-expiation ou le corps châtié, la punition-signe ou le corps marqué, la punition-exercice ou le corps dressé, la punition-bannissement ou le corps évincé), la modernité punitive se caractérisant par l'exclusion. Dans sa communication à Cerisy, Prairat repère dans la moyenne durée de l'histoire punitive de l'école (de la fin $\mathrm{du} \mathrm{XvI}^{\mathrm{e}}$ siècle à la réforme de juillet 2000) un "lent mouvement de libéralisation" (p.93) qu'il périodise en trois temps, celui de la rationalisation (1550-1800), celui de la libéralisation (1800-1960), celui des doutes (1960-2000), auxquelles périodes il fait correspondre trois grandes épistémès disciplinaires, l'épistémè religieuse (le salut), l'épistémè militaire (l'ordre) et enfin de nos jours, avec l'entrée du droit au cœur de l'école, l'épistémè politique (les dispositifs de régulation de la vie de classe et de la vie des établissements).

7 Un troisième groupe de textes étudie des phénomènes éducatifs du point de vue d'une durée plus courte, celle de l'institution républicaine (Prost, Vergnioux, Delalande, Largeaud, Manesse, Kahn). On peut même parler de très courte durée pour les textes de Houssaye, Best et Peyronie. En choisissant une durée de deux siècles, A. Prost rend visible deux grands moments de reconfiguration de l'ensemble du système scolaire. Il distingue trois périodes: l'âge des notables (avant 1879), l'âge républicain et l'âge 
démocratique. «L'enseignement français a bougé dans son ensemble deux fois, et deux fois seulement en deux siècles : avec les républicains, de 1879 à 1902, et avec la Cinquième République, de 1959 à 1985. » (p. 29). A. Prost montre aussi que dans les deux cas les réformateurs ont buté sur la même limite (la résistance de la pédagogie traditionnelle) et échoué à réaliser leurs objectifs de rénovation pédagogique et de formation des maîtres.

8 P. Kahn analyse, lui aussi, dans sa communication, comment, sous la Troisième République, la réforme pédagogique se heurte à la résistance de la forme scolaire héritée des frères des Écoles chrétiennes. En prenant en compte une durée de l'ordre du siècle, il montre que la modernité pédagogique liée à l'individualisme démocratique (la reconnaissance de l'élève comme enfant et de l'enfant comme individu) ne date pas de l'émergence de l'Éducation nouvelle dans les années 1910-1920 mais qu'elle remonte au «moment Buisson » (p. 189); il met ainsi en question une thèse commune aussi bien à M. Gauchet et P. Raynaud qu'à F. Dubet, à savoir l'idée que l'école deviendrait individualiste et démocratique en cessant d'être républicaine. Dans les discours de J. Ferry et surtout dans la première édition du Dictionnaire de pédagogie de Buisson, on peut repérer la constitution d'un discours puérocentrique. Il y a par conséquent une certaine continuité entre le discours pédagogique des républicains de la Troisième république et celui des pédagogues de l'Éducation nouvelle. La nouveauté de l'Éducation nouvelle réside plutôt dans le refus du modèle didactique de la transmission et du mode simultané d'organisation pédagogique, les républicains défendant une conception objectiviste du savoir qui exclut la discussion et acceptant la forme scolaire soit comme une contrainte inévitable (F. Buisson) soit comme un principe légitime (G. Compayré). Si donc la réforme pédagogique s'inscrit dans une certaine longue durée, la forme scolaire relève cependant d'une durée encore plus longue.

9 F. Best, sur une durée plus courte (de la Libération à la loi d'orientation de 1989), montre que l'Éducation nouvelle s'insère durablement dans l'institution scolaire ellemême et "résiste au temps et aux attaques dont elle est régulièrement l'objet " (p. 193). Elle prend comme exemples des pédagogues et hauts fonctionnaires qui ont été des artisans d'une réforme en douceur de l'Éducation Nationale: G. Monod et L. Cros (qui inventent en 1945 le dispositif des "classes nouvelles»), L. Legrand et quelques inspecteurs généraux comme J. Leif et $\mathrm{M}$. Rouchette (auxquels nous ajouterons le nom de F. Best elle-même). Mais chaque fois qu'un ministre veut généraliser un principe ou un dispositif qui est dans le droit fil de l'Éducation nouvelle, il se heurte à des oppositions insurmontables. "Autrement dit l'insertion de l'Éducation nouvelle dans l'institution scolaire se fait lentement et par à-coups. Il s'agit d'une évolution en courbe sinusoïdale et pas continue et rectiligne » (p. 198).

10 A. Vergnioux étudie le musée moderne entre la Révolution française (le musée républicain) et la Troisième République (le musée scolaire). «L'idée moderne du musée se dégage au début $d u$ XIx ${ }^{e}$ siècle de ses origines religieuses (le trésor des églises) et privées (le cabinet de curiosités) pour devenir un espace ouvert, démocratique " (p.69). Vergnioux distingue trois périodes: la période révolutionnaire, le moment Guizot et le moment ferryste. La Révolution française marque une rupture ; elle détruit du passé ce qui représente le pouvoir royal et le pouvoir religieux, mais en même temps elle conserve les œuvres et monuments en les exposant dans l'espace public à des fins de régénération et d'instruction du peuple. Avec Guizot, le musée participe, 
parallèlement à la généralisation de l'instruction primaire (loi de 1833), à la constitution d'une mémoire commune au service de la réconciliation nationale. "C'est la mise en place d'un système d'instruction élémentaire généralisé sur tout le territoire, c'est la diffusion au plus grand nombre, par des institutions contrôlées par l'État, d'une culture artistique et historique constituée en patrimoine commun.» (p. 80). Le château de Versailles est ainsi transformé par Louis-Philippe en un musée historique qui intègre la Révolution et l'Empire dans l'histoire de France. Enfin, sous la Troisième République, les musées pédagogiques et les musées scolaires (supports de la leçon de choses) sont, avec l'institution scolaire, les instruments de la diffusion de la culture scolaire républicaine.

11 Autre espace étudié : la cour de récréation. Dans la première partie de sa communication, J. Delalande traite du regard que l'institution porte sur cet espace depuis le XIX siècle; elle montre que dans cette période l'enfant globalement n'est pas reconnu dans ses capacités internes à se développer par lui-même et que les programmes officiels sont pris dans une contradiction (qui perdure aujourd'hui encore) de préconiser pour les enfants en récréation des activités à la fois libres et dirigées. Dans une deuxième partie elle étudie les points de vue contemporains sur la récréation. Si les textes officiels traitent surtout de l'aménagement de la cour, les ouvrages pédagogiques invitent les enseignants à en faire un moment éducatif. Dans les deux cas, les discours restent prisonniers d' "une perception dévalorisante des relations entre enfants » (p. 129). Au contraire, J. Delalande, d'un point de vue d'ethnologue inspiré par les travaux du folkloriste Van Gennep, est attentive à l'expérience et à la culture enfantines : les interactions entre enfants amènent ceux-ci à développer des éléments structurant le comportement individuel sur la base de principes de justice. Si le point de vue des enfants eux-mêmes était pris en compte (en particulier dans la formation des maitres), la relation entre enfants et adultes pourrait en être changée.

12 J.-M. Largeaud étudie l'histoire de l'enseignement de Waterloo. Cette bataille est en effet un événement de longue durée dans la culture française; elle a résisté à la désacralisation $\mathrm{du}$ patriotisme $\mathrm{au} \mathrm{xx}^{\mathrm{e}}$ siècle même si elle a perdu de sa force symbolique. J.-M. Largeaud montre que « seuls les républicains ont fait du 18 juin 1815, à partir de la Troisième République, un élément de leur éducation à la patrie fondée en morale.» (p. 150). La réflexion des républicains sur Waterloo est une conséquence du coup d'état de 1851 et de ce que celui-ci révèle de force du bonapartisme dans le peuple. Les républicains font de cette bataille un instrument de formation du citoyen, en dissociant le destin de la patrie (l'héroïsme français) de celui de Napoléon, dont le despotisme et la dérive guerrière sont fustigés. «L'interprétation républicaine n'est pas exempte de contradictions et porte la trace d'ambivalences parfois redoutables. Un des aspects de la synthèse républicaine réside en effet dans les contradictions entre le rejet du césarisme napoléonien et la fascination de Napoléon comme chef victorieux, défenseur malheureux de la patrie en 1815, entre le refus du militarisme et l'admiration pour les combattants de 1815. » (p. 147). Après 1919, Waterloo (désormais supplanté par Verdun) devient peu à peu une bataille comme une autre. Mais l'enseignement de la littérature française (Hugo, Stendhal) vient au secours de celui de l'histoire pour assurer la permanence de Waterloo dans la culture française.

13 La communication de D. Manesse porte sur la dictée (et on peut regretter que ce colloque n'ait pas traité d'autres exercices scolaires de longue durée). Née bien avant l'école de la République mais généralisée autour de 1880, cette pratique, qui a fait dès 
l'origine (J. Ferry et L. Bourgeois en ont dénoncé les dérives) et fait encore l'objet de débats, perdure depuis un siècle et demi. Cette persistance s'explique, selon D. Manesse, par l'ambiguïté qui caractérise la dictée. Rituel simple et sécurisant ou mise en scène dramatique et violente? Exercice d'évaluation ou d'apprentissage de l'orthographe? Enfin, si les pratiques d'enseignement de l'orthographe ont évolué, on peut penser cependant que les efforts pour aider les élèves à construire de façon réflexive le système orthographique, ne peuvent venir à bout de la dictée du fait de l'irrationalité d'une partie de ce système. "On peut difficilement penser l'enseignement de l'orthographe sans un accompagnement d'exercices systématiques [...] La dictée est un moyen de mettre en œuvre ces entraînements. » (p. 181). En outre elle semble irremplaçable comme outil collectif d'évaluation des élèves.

14 En prenant pour point de départ le numéro de la Revue française de pédagogie sur «Évaluer et comprendre les effets des pratiques pédagogiques » $\left(n^{\circ} 148,2004\right)$ puis en s'appuyant sur l'analyse des recherches consacrées aux bons et mauvais élèves et professeurs, J. Houssaye identifie des postures, des "humeurs épistémologiques» (p.151), sous-jacentes à la psychologie et à la sociologie en sciences de l'éducation depuis la deuxième moitié du $\mathrm{Xx}^{\mathrm{e}}$ siècle. "Il y a des traditions dans les postures de recherche [...] Être chercheur en... revient à adopter ces postures » (p. 156). Houssaye montre que la posture de la psychologie est optimiste et adaptatrice alors que celle de la sociologie est pessimiste et dénonciatrice. Dans les années dix-neuf cent (avec Claparède et Binet), la psychologie est applicationniste et cette posture va dominer la recherche (à l'exception de la psychologie clinique, pessimiste et dénonciatrice) tout au long de la deuxième moitié du siècle, même si certains chercheurs se montrent prudents quant au passage de la connaissance scientifique à la prescription normative. Pour ce qui est de la sociologie, c'est essentiellement dans les années soixante-dix qu'elle prend majoritairement une posture de dénonciation des mécanismes de discrimination sociale; mais dans les années quatre-vingt-dix, les sociologues tendent de plus en plus à proposer en vue d'améliorer et non plus seulement à dénoncer. J. Houssaye constate donc que «le descriptif et le prescriptif, l'explicatif et le normatif n'ont cessé de constituer les sciences de l'éducation dans leur définition, leur développement et leur fonctionnement. » (p. 166).

La communication d'H. Peyronie porte sur la coupure entre les mondes scolaires du primaire et du secondaire-supérieur : « comment, sur le fond de la résistance de cette coupure aux mutations successives [...] adviennent cependant des déplacements et des ruptures.»(p. 201). Peyronie se réfère dans un premier temps aux travaux des sociologues (V. Isambert-Jamati \& F. Muel-Dreyfus) qui ont analysé dans les années quatre-vingt la coupure entre les deux corps d'enseignants comme coupure à la fois professionnelle, sociale, culturelle et symbolique. Dans un deuxième temps, il met en évidence des déplacements et ruptures qui affectent cette coupure persistante. Il analyse d'abord une tentative, et l'échec de cette tentative, de collaboration entre des instituteurs militants du mouvement Freinet et des universitaires ou des cadres de l'administration scolaire à travers la revue Techniques de vie entre 1959 et 1962: dans cette expérience, ce sont les instituteurs qui maintiennent la coupure. Puis H. Peyronie analyse les transformations du métier d'enseignant dans les années quatre-vingt-dix où la création des IUFM suscite chez certains enseignants du secondaire et du supérieur un rejet du principe de la formation dans une même institution des professeurs du primaire et du secondaire. Il fait l'hypothèse que, si la détestation du primaire est en régression, elle s'est déplacée (au sens freudien du terme) sur le refus de la 
professionnalisation des enseignants. Cependant, d'un autre côté, les recompositions identitaires tendent à rapprocher les professeurs du secondaire et les professeurs des écoles. À l'échelle d'un siècle et demi, « le constat est contradictoire : les continuités tenaces coexistent avec les ruptures surprenantes. » (p. 217).

Il résulte de ce colloque une complexification des phénomènes scolaires. L'école est compliquée car elle est empêtrée dans des temporalités hétérogènes. En fin de compte elle apparaît, nous semble-t-il, comme étant prise dans les grands mouvements de modernisation démocratique de la société (sécularisation, libéralisation, individualisation) mais aussi comme ayant sa propre longue durée (ses modes spécifiques de transmission, ses exercices spécifiques, etc.). Il y a une contemporanéité et une non-contemporanéité de l'institution scolaire. Et une ambiguïté de cette noncontemporanéité : si, par certains côtés, l'école résiste à la modernité, par d'autres côtés et en tant qu'institution de la modernité, elle résiste aux effets dissolvants du postmodernisme.

\section{AUTEURS}

JEAN-MARC LAMARRE

IUFM des Pays de Loire 